\title{
The example of CaPSURE: lessons learned from a national disease registry
}

\author{
Sima P. Porten • Matthew R. Cooperberg • \\ Badrinath R. Konety $\cdot$ Peter R. Carroll
}

Received: 21 December 2010/ Accepted: 2 February 2011/Published online: 24 February 2011

(C) The Author(s) 2011. This article is published with open access at Springerlink.com

\begin{abstract}
Introduction Although randomized controlled trials (RCTs) remain the gold standard for determining evidence-based clinical practices, large disease registries that enroll large numbers of patients have become paramount as a relatively cost-effective additional tool.

Methods We highlight the advantages of disease registries focusing on the example of prostate cancer and the Cancer of the Prostate Strategic Urologic Research Endeavor $\left(\mathrm{CaPSURE}^{\mathrm{TM}}\right)$ registry.

Results CaPSURE collects approximately 1,000 clinical and patient-reported variables, in over 13,000 men that are enrolled. Thus far, CaPSURE has yielded over 130 peerreviewed publications, with several others in press, in key areas of risk migration, practice patterns, outcome prediction, and quality of life outcomes.

Conclusions Disease registries, like CaPSURE complement RCTs and CaPSURE, have provided a means to better understand many aspects of prostate cancer epidemiology, practice patterns, oncologic and HRQOL outcomes, and costs of care across populations. Specialized observational disease registries such as CaPSURE provide
\end{abstract}

S. P. Porten - M. R. Cooperberg · P. R. Carroll Department of Urology, UCSF Helen Diller Comprehensive Cancer Center, University of California,

San Francisco, CA, USA

B. R. Konety

Department of Urology, University of Minnesota,

Minneapolis, MN, USA

P. R. Carroll ( $\square)$

UCSF/Mt. Zion Cancer Center, 1600 Divisadero St,

3rd Floor, San Francisco, CA 94115-1695, USA

e-mail: pcarroll@urology.ucsf.edu insight and have broad implications for disease management and policy.

Keywords Prostatic neoplasms - CaPSURE - Disease registries

\section{Introduction}

The randomized controlled trial (RCT) remains the gold standard for informing evidence-based clinical practice, in urology as in other medical domains. Limitations include time, significant expense, stringent inclusion criteria, and resistance to randomization by clinicians and patients. Ultimately, if patients enrolled in a RCT differ from the larger population of patients with a given condition, the external validity of the findings may be questionable. Although these limitations can be mitigated by utilizing specialized RCT designs (Table 1), disease registries have emerged as an important complement to RCTs. Disease registries, which accrue prospectively identified cohorts and follow them regardless of sociodemographic characteristics, clinical variables, treatment details or intermediate outcomes, have emerged as an important complement to RCTs. This reflection of "real world" treatment is a tremendous asset especially in prostate cancer research as it provides a relatively costeffective tool to shed light on a disease with a long natural history and rapidly changing management practices that are subject to many different clinical, scientific, demographic, and economic dynamics. This article will highlight some advantages of disease registries focusing on the example of prostate cancer and the Cancer of the Prostate Strategic Urologic Research Endeavor $\left(\right.$ CaPSURE $\left.^{\mathrm{TM}}\right)$ registry. 
Table 1 Specialized classifications of randomized controlled trials

\begin{tabular}{|c|c|c|c|}
\hline Type & Description & Strengths & Limitations \\
\hline Cluster & $\begin{array}{l}\text { Randomization of subjects as a group } \\
\text { rather than on individual basis }\end{array}$ & $\begin{array}{l}\text { Can study interventions that cannot be directed } \\
\text { toward selected individuals } \\
\text { Can control for contamination across } \\
\text { individuals (i.e., when one individual's } \\
\text { behavior can influence another's) }\end{array}$ & $\begin{array}{l}\text { Needs more subjects to reach statistical } \\
\text { power than standard RCT }\end{array}$ \\
\hline Explanatory & $\begin{array}{l}\text { Individual randomization of very } \\
\text { selective subjects in a highly } \\
\text { controlled setting }\end{array}$ & $\begin{array}{l}\text { Useful to test efficacy (i.e., whether an } \\
\text { intervention causes a specific biologic } \\
\text { response) } \\
\text { Patients blinded } \\
\text { Excellent internal validity } \\
\text { Good for acute disease processes }\end{array}$ & $\begin{array}{l}\text { External validity and applicability to } \\
\text { clinical practice is limited due to subject } \\
\text { selection }\end{array}$ \\
\hline Pragmatic & $\begin{array}{l}\text { Individual randomization of non- } \\
\text { selective group of patients in a } \\
\text { regular clinical setting }\end{array}$ & $\begin{array}{l}\text { Useful to test effectiveness of an intervention } \\
\text { in everyday practice } \\
\text { Good for chronic disease processes and } \\
\text { complex interventions } \\
\text { Excellent external validity and directly } \\
\text { applicable to clinical practice }\end{array}$ & $\begin{array}{l}\text { Patients unblinded } \\
\text { Internal validity limited due to broad } \\
\text { inclusion criteria }\end{array}$ \\
\hline $\begin{array}{l}\text { Expertise- } \\
\text { based }\end{array}$ & $\begin{array}{l}\text { Individual randomization of subjects } \\
\text { to an expertise in the intervention } \\
\text { in question }\end{array}$ & $\begin{array}{l}\text { Useful when intervention is non-pharmacologic } \\
\text { (i.e., surgical procedures) }\end{array}$ & $\begin{array}{l}\text { External validity limited to only those } \\
\text { patients receiving care from a physician } \\
\text { with expertise skills }\end{array}$ \\
\hline
\end{tabular}

CaPSURE was initiated in 1995 to document national trends in prostate cancer epidemiology, disease management, oncologic outcomes, and health-related quality of life (HRQOL) outcomes. It is a longitudinal, observational database accruing data from a total of 40 urologic practice sites over the history of the registry. The majority of sites are community based, although four university-affiliated centers, and Veterans Affairs (VA) medical centers are included. Men with biopsy-proven cancer are invited to join CaPSURE regardless of disease stage or treatment history. Informed consent for participation is obtained under institutional review board supervision.

CaPSURE collects approximately 1,000 clinical and patient-reported variables. Clinical information is collected by the treating urologist at baseline and with each followup visit and includes history of prostate cancer diagnosis, biopsies, pathology, staging tests, primary and subsequent treatments (radical prostatectomy [RP], external beam radiotherapy [EBRT], brachytherapy, primary and neoadjuvant androgen deprivation therapy [PADT and NADT], cryosurgery, and watchful waiting/active surveillance [AS]), Karnofsky performance status scores, clinic procedures, and medications. At enrollment and every 6 months thereafter, a questionnaire is completed documenting sociodemographic parameters, comorbidities, and HRQOL using validated instruments. Other sections of the patient questionnaires assess use of health services, with hospitalization data verified by discharge summary review.

Patients are treated according to their physicians' usual practices and patient preferences and are followed until time of death or withdrawal from the study. Periodic, random sample chart review ensures completeness and accuracy of data collected and entered. Additional details regarding project methodology have been reported previously [1]. CaPSURE is managed by the Department of Urology at the UCSF Helen Diller Family Comprehensive Cancer Center. It was funded from inception to 2007 through an unrestricted education grant from TAP Pharmaceutical Products, Inc., and currently is supported through Abbott Labs (Chicago, IL) and several collaborative Federal grants.

There are currently 13,821 men enrolled in CaPSURE. The median patient age at diagnosis is 67 , and $75 \%$ of men are between 60 and 79 years of age. Most patients are white, with approximately $10 \%$ black representation, and $3.5 \%$ Latino, Asian, and other ethnicities. There is a fairly even distribution across socioeconomic strata, based on education and income level. CaPSURE has yielded over 130 peer-reviewed publications, with several others in press. A summary of some key research findings follows.

\section{Trends in prostate cancer presentation and risk}

Temporal trends in the PSA era in patient risk at diagnosis are consistent with downward stage migration. In $\mathrm{CaP}$ SURE, the proportion of patients presenting with low-risk disease $(\mathrm{PSA}<10 \mathrm{ng} / \mathrm{mL}$, Gleason score $<7$ with no pattern 4 or 5 disease on biopsy, and clinical stage T1 or T2 disease) has increased from $31 \%$ between 1989 and 1990 to $47 \%$ of patients between 2001 and 2002 and has remained 
relatively stable [2]. However, within this low-risk group, there is a trend toward lower-risk characteristics (based on PSA, clinical stage, and percent positive biopsy). Conversely, during the same time, men with high-risk disease (PSA $>20 \mathrm{ng} / \mathrm{mL}$, Gleason 8-10 on biopsy, or stage T3) have decreased from 41 to $29 \%$. Although the rate of highrisk prostate cancer has fallen, it has remained stable since 2000 and represents approximately $24 \%$ of patients in recent studies [3]. There is no evidence in the CaPSURE cohort of meaningful downward risk migration among high-risk patients over the past 15 years.

Despite presentation with lower-risk disease due to increased PSA screening, recent studies have observed a disparity in prostate cancer presentation across sociodemographic groups. Dall'era et al. [4] examined 5,939 men enrolled in CaPSURE from 1995 to 2007, and found that patients who were older, less educated, and had Medicare for insurance (as opposed to VA or private coverage) were more likely to have intermediate or high-risk disease. Nonwhite race was also associated with high-risk disease at presentation (OR 1.83, 1.47-2.29, $P<0.01$ ). Clinically insignificant disease (PSA $<10 \mathrm{ng} / \mathrm{mL},<33 \%$ of biopsy cores involved, no Gleason pattern $4 / 5$, and stage T1a or T2a) was more common in younger men $(<60$ years old), higher income/education, and men with private insurance. Within this group, younger age and private insurance were again associated with immediate treatment in lieu of AS.

\section{Health services research and trends in disease management}

The majority of patients followed in CaPSURE were diagnosed during the PSA era and treated in communitybased settings. One strength of this registry is that participating physicians treat according to their usual practices and patient preferences. CaPSURE provides a mix of locales and practice types, reflecting current contemporary urological practice at a national level. The CaPSURE sites are not a random sample of the US population. However, CaPSURE includes far richer clinical detail than population-based sources such as the Surveillance Epidemiology and End Results (SEER) database and SEER-Medicare and therefore is an excellent data source for national studies of disease management.

Imaging studies performed in men with prostate cancer may serve to facilitate optimal treatment planning. However, staging studies are associated with low but definite risks, significant costs to the health care system, and have minimal benefit in patients with low-risk disease characteristics. One early study from CaPSURE examined the use of imaging tests for staging clinically localized prostate cancer between 1989 and 1997. Kindrick et al. [5] found widespread and consistent overuse in the rates of bone scan, computed tomography, and magnetic resonance imaging among patients with low likelihood of extraprostatic disease. Follow-up analysis through 2001 showed that rates of bone scan and computerized tomography use have decreased in recent years with the greatest decreases in patients with lower-risk cancer [6]. Whereas among early CaPSURE patients, disease risk exerted no influence on the likelihood of imaging, the more recent CaPSURE data have illustrated a trend toward appropriate and evidence-based use of imaging tests. This highlights the value of registries such as CaPSURE that allows evaluation of adherence to guidelines based on high-level evidence into common clinical practice, as well as the extent to which evidencebased medicine is practiced in urology.

Multiple recent CaPSURE studies have examined patterns in treatment selection for patients diagnosed with prostate cancer. An early study examined the use of ADT in patients with localized disease and found a higher than expected use of ADT monotherapy [7]. Among low-, intermediate-, and high-risk patients, ADT monotherapy rose dramatically, from 5 to $14 \%, 9$ to $20 \%$, and 33 to $48 \%$, respectively, from 1989-1990 to 2000-2001. PADT monotherapy is considered to be investigational based on the American Urological Association's clinical practice guidelines [8], and no controlled trials have established efficacy of this approach.

With decreasing risk migration, it would be expected that the use of AS would increase since more men are presenting with favorable risk disease. An early study of temporal trends found that the use of AS reached a nadir of $5.5 \%$ in $2000-2001$ from $9.5 \%$ in 1992-1994, with the largest declines in low-risk patients (6.2\%), although recent improvement to $10.2 \%$ in 2004-2006 was observed [2]. Another study by Barocas et al. [9] found that between 1999 and 2004, 16.4\% of men met strict surveillance criteria (PSA $<10 \mathrm{ng} / \mathrm{mL}$, clinical T1 or T2a, prostate-specific antigen density PSAD $<0.15,<33 \%$ biopsy cores positive, and absence of Gleason pattern $4 / 5$ on biopsy), but only $9 \%$ of men in this low-risk category chose surveillance, highlighting the underuse of this management strategy in the United States.

In addition to the initial decline in AS, a decrease was observed in EBRT from 13\% in 1993-1995 to 7\% in 1999-2001 and RP (55-52\%) and increases in PADT (7-12\%) and brachytherapy (4-22\%) in low-risk patients [10]. This treatment trend remained present in men $>75$ years with the use of surveillance in only $24 \%$, declining from $52 \%$, PADT use increasing from 23 to $30 \%$, and brachytherapy from 3 to $31 \%$. A reversal of this trend was observed with a decrease in brachytherapy to $13 \%$ in 2005-2006, a decrease in PADT to 6.6\%, and an increase in RP to $60 \%$ [2]. 
Overall, it appears that prostate cancer risk drives treatment selection in conjunction with age, comorbidity, and socioeconomic status. However, a recent analysis in CaPSURE examining practice patterns in the primary treatment of localized prostate cancer confirmed prevalent overtreatment of low-risk disease but also identified what appeared to be a troubling undertreatment of high-risk disease $[3,11]$. Interestingly, treatment patterns were found to vary across clinical sites, and much of the variation could be attributed to practice site itself and not solely patient or tumor factors. Clinical practice site alone explained, for example, 13\% variation seen in ADT, 30\% in RP, $36 \%$ in brachytherapy, $20 \%$ in EBRT, and $74 \%$ in cryoablation [11].

Another unique feature of CaPSURE is the access to resource use data that offer a means of studying health care cost implications of different prostate cancer management strategies. Penson et al. [12] examined adjusted first-year costs associated with various treatment options based on Medicare payment schedules and found a trend toward higher costs for higher-stage patients. The mean cost of prostate cancer treatment in the first year after diagnosis was $\$ 6,375$ and was not different between patients with RP and EBRT, but was higher for those receiving NADT prior to either treatment. Wilson et al. [13] analyzed all health care utilization of various treatments over a period of 5 years and found that the average annual cost was $\$ 7,740$. This varied widely with AS costing the least at \$5,843 and androgen deprivation therapy the most expensive at $\$ 12,590$.

\section{Oncologic outcomes}

Recent evidence suggests that information beyond Gleason score obtained at diagnostic biopsy contributes significantly to accurate risk assessment among patients with newly diagnosed prostate cancer. The percent of positive biopsy cores was initially validated as a prognostic marker among patients who underwent RP in CaPSURE and was significant across all risk groups, confirming that biopsy data obtained in the community setting using non-standardized techniques and assessed by diverse pathologists offers consistent and useful prognostic information [14].

Cancer of the Prostate Risk Assessment score (CAPRA) was developed using data from the CaPSURE registry. The CAPRA score simply and accurately predicts pathologic status, disease recurrence, and mortality after surgery [15]. Points are assigned based on age, clinical stage, PSA, Gleason grade, and percent of cores positive on biopsy.
Scores range from 0 to 10 with each 2-point increase roughly doubling the risk of recurrence and progression. A 9-point variation of the CAPRA scoring system can alternatively be used if data regarding percent positive biopsy cores are not available. The strength of the CAPRA score is not only in its ease of use but in its ability to better discriminate between categories of risk in all practice settings when compared with other nomograms [16, 17]. The CAPRA score has been extensively validated in other disease registries and academic cohorts, both in the United States and Europe [18].

Uncertainty regarding the optimal treatment for localized prostate cancer has led to large variation in practice patterns as reported previously in CaPSURE [11]. To date, no adequate randomized trials have compared active treatments for localized prostate cancer due to difficulties with accrual, randomization, high cost, and need for long follow-up. Disease registries can provide important insights into outcomes and can provide an important source for comparative effectiveness analysis between various treatments. This is highlighted in a recent study by Cooperberg et al. [19] which compared risk-adjusted diseasespecific and all-cause mortality after treatment of localized prostate cancer with RP, EBRT, and primary ADT. After adjusting for age, disease risk, and comorbidity, mortality at 10 years was less likely in men who underwent RP than EBRT or primary ADT, especially in men with intermediate or high-risk disease.

\section{Quality of life outcomes}

The preservation of health-related quality of Life (HRQOL) is a priority in any discussion of prostate cancer treatment and outcomes due to the long natural history of disease. Any negative impact on quality of life must be minimized, as patients may experience it for an extended period of time. CaPSURE had proved to be an invaluable resource for the prospective, longitudinal assessment of patient-reported outcomes and it has enabled investigators to successfully address a number of questions in this area of prostate cancer research.

CaPSURE HRQOL data are reported by both patients and physicians and provide insight into the differences between outcomes. An early study by Litwin et al. [20] found that physician assessment of treatment impact on HRQOL underestimated the impact experienced by patients, especially within general health parameters. When impairment was noted, urologists reported on urinary and sexual functions more often than pain in men who underwent treatment for localized prostate cancer. Many aspects 


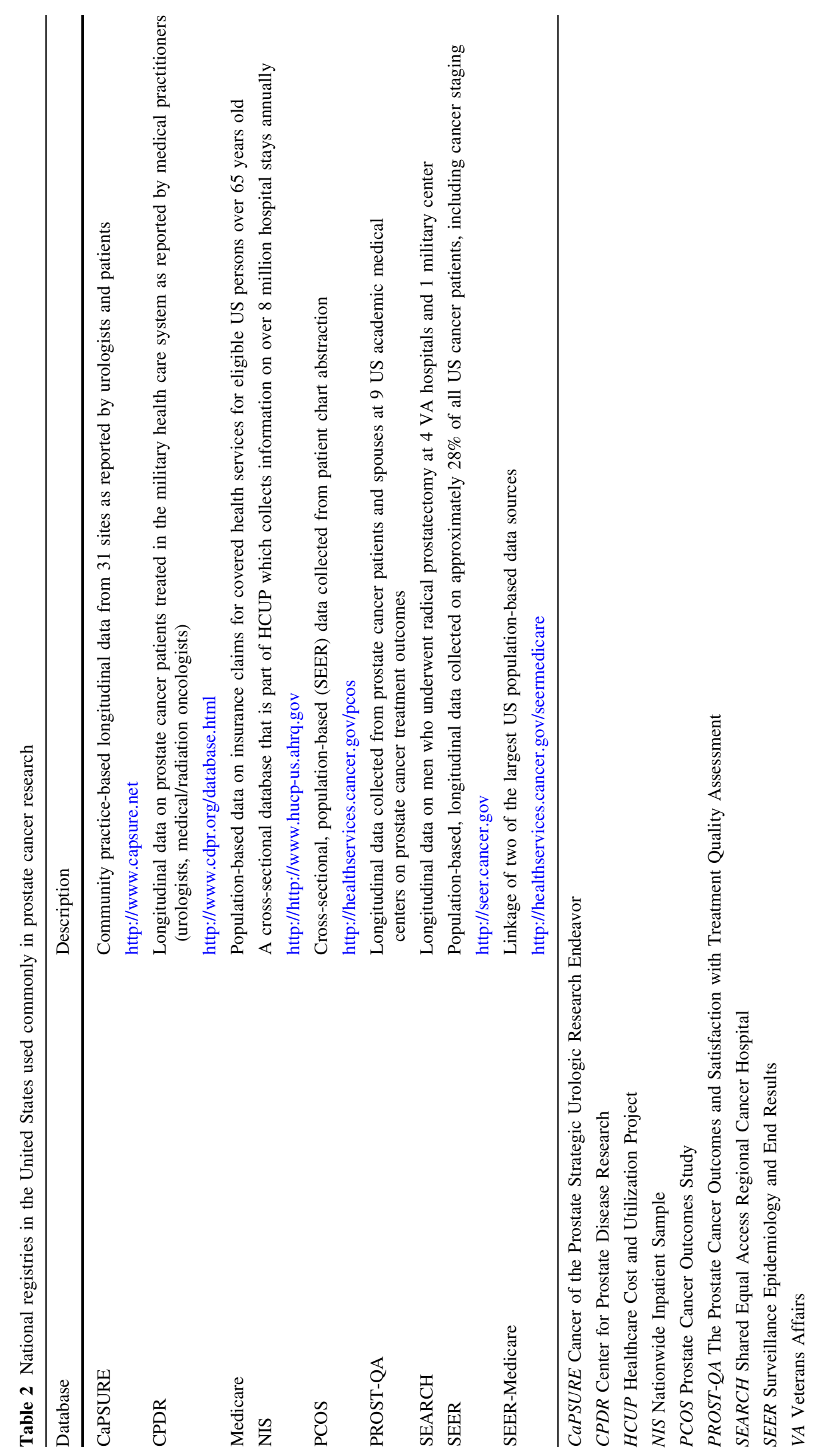


of patient-reported HRQOL (physical function and general health) have also been significantly associated with survival in a similar cohort of men, over the course of disease from diagnosis to after treatment [21]. These studies highlight the continued importance of multidimensional, patient-reported HRQOL assessment in current prostate cancer treatment.

Using longitudinal HRQOL data within CaPSURE, comparisons can be made between outcomes among treatment options. Men who underwent RP had lower scores on both disease-specific and general HRQOL instruments immediately postoperative, which improved significantly at 1 year after treatment, and continued to improve in the domain of sexual function in the second year. Men who were treated with EBRT, AS, or primary ADT had scores that were relatively stable, except for sexual function, which declined with time. Overall, patients who underwent RP had the greatest decline initially, but also the greatest degree of recovery. Most men experienced the greatest recovery of both urinary and sexual functions within 2 years of treatment, with little change in reported HRQOL scores after 3 years [22]. Those who received multimodal therapy appeared to have greater declines in urinary and sexual functions than those who were treated with monotherapy [23].

Although prostate cancer presentation and treatment patterns vary based on ethnicity, little was known with regards to any differences in HRQOL. Disease registries like CaPSURE are ideal to study ethnic groups that are underrepresented in most clinical studies. Using CaPSURE data, African American men were found to have lower HRQOL scores across many domains of QOL at baseline and after treatment, but had higher sexual function scores [24]. Over time, African American patients never recovered as well as white patients in reported HRQOL scores. Ethnicity was also found to play a role in primary treatment choice in men with equivalent disease characteristics [25].

Although this article focuses primarily on lessons learned from CaPSURE, there are a number of other US national registries and databases available for research. Table 2 highlights the strengths of many of the resources available to study the various aspects of prostate cancer. For example, the Prostate Outcomes Cancer Study (PCOS), which utilizes Surveillance Epidemiology and End Results (SEER)-based data, was initiated by the National Cancer Institute (NCI) and used by investigators to report on HRQOL outcomes in American men diagnosed with prostate cancer [26]. Likewise, the Department of Defense Center for Prostate Disease Research (CPDR) has been used to report on prostate cancer-specific mortality and not only collects data on men with prostate cancer treated within the military medical system but seeks to standardize clinical practice among military sites [27].

Limitations to disease registries in general center on methodology of data collection. The quality of data collected and integrity in data collection and follow-up determine the quality of the disease registry. In addition to bias introduced by data collection, disease registries do not capture a random sample of the population. These biases can be minimized (as in CaPSURE) by including community centers to increase population sampling. Centralizing data collection and analysis with strict monitoring by a statistician ensure quality control. Although CaPSURE does capture a diverse group of men, patients are enrolled by their urologists, rather than medical or radiation oncologists, which may exclude a proportion of men with prostate cancer.

CaPSURE is a research partnership with industry (initially funded by TAP pharmaceuticals) under IRB approval. Data integrity has been maintained free of conflict of interest by thoughtful and transparent methodology and reporting. Data analyses and decision for publication of CaPSURE results have always rested with academic investigators without the influence of industry. Currently, CaPSURE is supported by a combination of gifted funds from Abbott Laboratories along with a growing portfolio of federal grants to ensure registry continuity and maintenance.

\section{Conclusion}

While RCTs remain the gold standard for advancing knowledge in medicine, they can be difficult to complete and expensive; moreover, in a disease with a prolonged natural history, treatment strategies may evolve quickly that even a well-executed trial may not be relevant by the time it is published. Disease registries, like CaPSURE, have provided a means to better understand many aspects of prostate cancer epidemiology, practice patterns, outcomes, and costs of care. It remains a robust source of information and provides a cost-effective way of driving evidence-based decisions regarding treatment and health policy. Specialized observational disease registries such as CaPSURE provide insight and have broad implications for disease management and policy.

Conflict of interest Drs. Cooperberg and Carroll are CaPSURE researchers. Drs. Porten and Konety have no disclosures.

Open Access This article is distributed under the terms of the Creative Commons Attribution Noncommercial License which permits any noncommercial use, distribution, and reproduction in any medium, provided the original author(s) and source are credited. 


\section{References}

1. Lubeck DP, Litwin MS, Henning JM, Stier DM, Mazonson P, Fisk R, Carroll PR (1996) The CaPSURE database: a methodology for clinical practice and research in prostate cancer. CaPSURE Research Panel. Cancer of the Prostate Strategic Urologic Research Endeavor. Urology 48(5):773-777. doi:S0090429596002269

2. Cooperberg MR, Broering JM, Kantoff PW, Carroll PR (2007) Contemporary trends in low risk prostate cancer: risk assessment and treatment. J Urol 178(3 Pt 2):S14-S19. doi:10.1016/j.juro. 2007.03.135

3. Cooperberg MR, Cowan J, Broering JM, Carroll PR (2008) Highrisk prostate cancer in the United States, 1990-2007. World J Urol 26(3):211-218. doi:10.1007/s00345-008-0250-7

4. Dall'era MA, Hosang N, Konety B, Cowan JE, Carroll PR (2009) Sociodemographic predictors of prostate cancer risk category at diagnosis: unique patterns of significant and insignificant disease. J Urol 181 (4):1622-1627; discussion 1627. doi:10.1016/j.juro. 2008.11.123

5. Kindrick AV, Grossfeld GD, Stier DM, Flanders SC, Henning JM, Carroll PR (1998) Use of imaging tests for staging newly diagnosed prostate cancer: trends from the CaPSURE database. J Urol 160(6 Pt 1):2102-2106

6. Cooperberg MR, Lubeck DP, Grossfeld GD, Mehta SS, Carroll PR (2002) Contemporary trends in imaging test utilization for prostate cancer staging: data from the cancer of the prostate strategic urologic research endeavor. J Urol 168(2):491-495. doi: S0022-5347(05)64665-4

7. Cooperberg MR, Grossfeld GD, Lubeck DP, Carroll PR (2003) National practice patterns and time trends in androgen ablation for localized prostate cancer. J Natl Cancer Inst 95(13):981-989

8. Thompson I, Thrasher JB, Aus G, Burnett AL, Canby-Hagino ED, Cookson MS, D'Amico AV, Dmochowski RR, Eton DT, Forman JD, Goldenberg SL, Hernandez J, Higano CS, Kraus SR, Moul JW, Tangen CM (2007) Guideline for the management of clinically localized prostate cancer: 2007 update. J Urol 177(6):2106-2131. doi:10.1016/j.juro.2007.03.003

9. Barocas DA, Cowan JE, Smith JA Jr, Carroll PR (2008) What percentage of patients with newly diagnosed carcinoma of the prostate are candidates for surveillance? An analysis of the CaPSURE database. J Urol 180 (4):1330-1334; discussion 13341335. doi:10.1016/j.juro.2008.06.019

10. Cooperberg MR, Lubeck DP, Meng MV, Mehta SS, Carroll PR (2004) The changing face of low-risk prostate cancer: trends in clinical presentation and primary management. J Clin Oncol 22(11):2141-2149. doi:10.1200/JCO.2004.10.062JCO.2004.10.062

11. Cooperberg MR, Broering JM, Carroll PR (2010) Time trends and local variation in primary treatment of localized prostate cancer. J Clin Oncol 28(7):1117-1123. doi:10.1200/JCO.2009.26.0133

12. Penson DF, Schonfeld WH, Flanders SC, Henke CJ, Warolin KL, Carroll PR, Litwin MS (2001) Relationship of first-year costs of treating localized prostate cancer to initial choice of therapy and stage at diagnosis: results from the CAPSURE database. Urology 57(3):499-503. doi:S0090-4295(00)01033-5

13. Wilson LS, Tesoro R, Elkin EP, Sadetsky N, Broering JM, Latini DM, DuChane J, Mody RR, Carroll PR (2007) Cumulative cost pattern comparison of prostate cancer treatments. Cancer 109(3):518-527. doi:10.1002/cncr.22433

14. Grossfeld GD, Latini DM, Lubeck DP, Broering JM, Li YP, Mehta SS, Carroll PR (2002) Predicting disease recurrence in intermediate and high-risk patients undergoing radical prostatectomy using percent positive biopsies: results from CaPSURE. Urology 59(4):560-565. doi:S0090429501016582
15. Cooperberg MR, Pasta DJ, Elkin EP, Litwin MS, Latini DM, Du Chane J, Carroll PR (2005) The University of California, San Francisco cancer of the prostate risk assessment score: a straightforward and reliable preoperative predictor of disease recurrence after radical prostatectomy. J Urol 173(6):1938-1942. doi:10.1097/01.ju.0000158155.33890.e7

16. Greene KL, Meng MV, Elkin EP, Cooperberg MR, Pasta DJ, Kattan MW, Wallace K, Carroll PR (2004) Validation of the Kattan preoperative nomogram for prostate cancer recurrence using a community based cohort: results from cancer of the prostate strategic urological research endeavor (capsure). J Urol 171(6 Pt 1):2255-2259. doi:00005392-200406000-00033

17. Penson DF, Grossfeld GD, Li YP, Henning JM, Lubeck DP, Carroll PR (2002) How well does the Partin nomogram predict pathological stage after radical prostatectomy in a community based population? Results of the cancer of the prostate strategic urological research endeavor. J Urol 167 (4):1653-1657; discussion 1657-1658. doi:S0022-5347(05)65172-5

18. May M, Knoll N, Siegsmund M, Fahlenkamp D, Vogler H, Hoschke B, Gralla O (2007) Validity of the CAPRA score to predict biochemical recurrence-free survival after radical prostatectomy. Results from a european multicenter survey of 1,296 patients. J Urol 178 (5):1957-1962; discussion 1962. doi: 10.1016/j.juro.2007. 07.043

19. Cooperberg MR, Vickers AJ, Broering JM, Carroll PR Comparative risk-adjusted mortality outcomes after primary surgery, radiotherapy, or androgen-deprivation therapy for localized prostate cancer. Cancer. doi:10.1002/cncr.25456

20. Litwin MS, Lubeck DP, Henning JM, Carroll PR (1998) Differences in urologist and patient assessments of health related quality of life in men with prostate cancer: results of the CaPSURE database. J Urol 159(6):1988-1992. doi:S0022-5347(01)63222-1

21. Sadetsky N, Hubbard A, Carroll PR, Satariano W (2009) Predictive value of serial measurements of quality of life on all-cause mortality in prostate cancer patients: data from CaPSURE (cancer of the prostate strategic urologic research endeavor) database. Qual Life Res 18(8):1019-1027. doi:10.1007/s11136-009-9526-7

22. Huang GJ, Sadetsky N, Penson DF (2010) Health related quality of life for men treated for localized prostate cancer with long-term followup. J Urol 183(6):2206-2212. doi:S0022-5347(10)00254-5

23. Wu AK, Cooperberg MR, Sadetsky N, Carroll PR (2008) Health related quality of life in patients treated with multimodal therapy for prostate cancer. J Urol 180 (6):2415-2422; discussion 2422. doi:10.1016/j.juro.2008.08.015

24. Lubeck DP, Kim H, Grossfeld G, Ray P, Penson DF, Flanders SC, Carroll PR (2001) Health related quality of life differences between black and white men with prostate cancer: data from the cancer of the prostate strategic urologic research endeavor. J Urol 166(6):2281-2285. doi:S0022-5347(05)65551-6

25. Moses KA, Paciorek AT, Penson DF, Carroll PR, Master VA (2010) Impact of ethnicity on primary treatment choice and mortality in men with prostate cancer: data from CaPSURE. J Clin Oncol 28(6):1069-1074. doi:10.1200/JCO.2009.26.2469

26. Penson DF, McLerran D, Feng Z, Li L, Albertsen PC, Gilliland FD, Hamilton A, Hoffman RM, Stephenson RA, Potosky AL, Stanford JL (2005) 5-year urinary and sexual outcomes after radical prostatectomy: results from the prostate cancer outcomes study. J Urol 173(5):1701-1705. doi:10.1097/01.ju.0000154637. $38262.3 \mathrm{a}$

27. D'Amico AV, Moul JW, Carroll PR, Sun L, Lubeck D, Chen MH (2003) Surrogate end point for prostate cancer-specific mortality after radical prostatectomy or radiation therapy. $\mathbf{J}$ Natl Cancer Inst 95(18):1376-1383 